\title{
Modeling Uncertainties in Proactive Routing Protocols for Ad Hoc Networks
}

\author{
Hanen Idoudi*’**, Miklos Molnar**, Abdelfatteh Belghith*, Bernard Cousin** \\ * National School of Computer Sciences, Manouba, Tunisia \\ **IRISA, Campus de Beaulieu, Rennes, France \\ \{hanen.idoudi,abdelfattah.belghith\}@ensi.rnu.tn \\ \{hidoudi,molnar,bcousin\}@irisa.fr
}

\begin{abstract}
In this paper we introduce a new model for ad hoc networks. Our model aims at reproducing the states alternation of links and nodes led by dynamic and random topology of ad hoc networks as well as random delays of packets delivery. Furthermore, we study the phenomenon of uncertainties in routing operation due to the unpredictable topological changes. Unlike mobility models which reflect only the impact of mobility on routing protocols, our proposal can demonstrates the impact of several identified hazards on the performances of proactive routing protocols. We show through various simulations the performances of our model within the framework of a proactive routing.
\end{abstract}

Index Terms-Ad hoc Networks, Mobility Models, Modeling, Proactive Routing.

\section{INTRODUCTION}

A $\mathrm{d}$ hoc networks are wireless networks deprived of any stable fixed Infrastructure, and central management entity. They suffer from the spontaneous appearance, disappearance and mobility of the composing nodes. This aspect implies a dynamic and randomly evolving topology in time and space.

Several networks models have been proposed. Few of them address ad hoc networks. Most are mobility models which try to reproduce only the mobility of nodes in order to generate ad hoc topologies.

Most studies carried out to analyze performances of ad hoc networks protocols are based on mobility models. However, these models are insufficient to model all risks affecting same ad hoc functionalities such as routing.

The routing function is distributed between all the nodes of the ad hoc network which can operate at any moment as source, destination or router. Each node has to build the paths it needs and may have to maintain routing tables. Routing protocols in ad hoc networks are commonly divided in 2 categories: proactive and reactive. The proactive class [1] [5] can be divided into two sub classes according to the method for the routing information distribution. The two principal methods used are: link state and distance vector. The two methods require a periodic update of the routing table maintained by each node of the network. This task is done by exchange of topological data between the various nodes of the network. The algorithms based on these two methods, can use the same technique to compute paths which is the shortest paths, and thus allow a given host, to find the next host to reach the destination by using the shortest path in term of hop number. Reactive routing protocols [1] [5] create and maintain paths only when needed. When a node needs a path, a discovery process is launched. Although several routing protocols were proposed [2][6][3][4], the majority adapt badly to specificities of the ad hoc network such as mobility, spontaneous appearances and disappearances of nodes and random delays

In this article, we show the existence of specific risks leading to routing uncertainties in ad hoc networks and we point out that existing network models don't reflect these risks. We propose as a solution, a new model for ad hoc networks, called MarkovNet. Our proposal, can model, in a simple way, the evolution over time of both link's and node's states. To prove the efficiency of our model, we point out by several simulations, that MarkovNet can demonstrate the significant uncertainty of routing information within the framework of a proactive routing as well as previous ad hoc models.

Our article is organized as follows. In the following section we give an overview of existing mobility models then we analyze the problem of the uncertainty of routing in ad hoc networks to identify the risks in ad hoc routing. Thereafter, we define our proposal: the MakovNet model and the context of our experiments. In section 4, we display the results of simulations which enabled us to highlight the behavior of our model with the presence of imprecise routing information.

\section{RELATED WORK}

\section{A. Mobility models}

Several network models have been proposed 
$[9][10][11][12]$. For ad hoc networks, mobility models are likely to be the most suitable to address such type of networks and mobile networks in general.

A mobility model is designed to describe the movement pattern of mobile nodes, and how their location, direction and speed change over time. Fig.1-1 provides a classification for various mobility models based on their specific mobility characteristics.

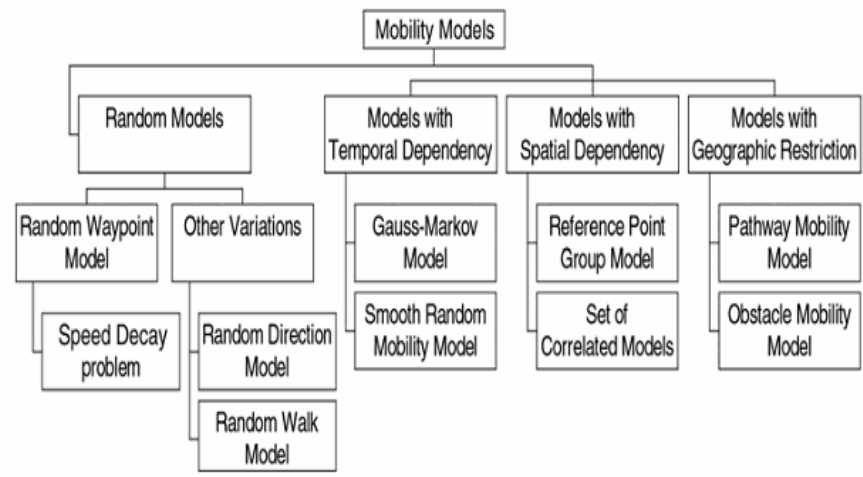

Fig. 1. Classification of mobility models

In random-based mobility models, the mobile nodes move randomly and freely without restrictions. Destination, speed and direction are all chosen randomly and independently of other nodes. One frequently used mobility model is the Random Waypoint model [13]. Each mobile node randomly selects one location as destination. It then travels towards this destination with constant velocity chosen uniformly and randomly from $[0, \mathrm{~V}]$, where $\mathrm{V}$ is the maximum allowable velocity for every mobile node [14]. The velocity and direction of a node are chosen independently of other nodes. Upon reaching the destination, the node stops for a duration defined by the 'pause time' parameter. After this duration, it chooses another random destination in the simulation field and moves towards it. Two variants of the Random Waypoint model, the Random Walk model and the Random Direction model were also proposed.

Mobility of a node may be constrained and limited by the physical laws of acceleration, velocity and rate of change of direction. Hence, the current velocity of a mobile node may depend on its previous velocity. Thus the velocities of single node at different time slots are 'correlated'. However, the memoryless nature of former mobility models renders them inadequate to capture the temporal dependency behavior existing in some mobility scenarios. As a result, various mobility models considering temporal dependency are proposed such as the Gauss-Markov Mobility Model [15] and the Smooth Random Mobility Model [17].

In random or temporal dependency based models, a mobile node moves independently of other nodes, the location, speed and movement direction of mobile node are not affected by other neighboring nodes. However, in some targeted MANET applications including disaster relief and battlefield, team collaboration exists and users are likely to follow the team leader. Therefore, the mobility of a mobile node could be influenced by other neighboring nodes. Since the velocities of different nodes are 'correlated' in space, thus we call this characteristic as the Spatial Dependency of velocity.

Reference Point Group Mobility (RPGM) Model is proposed in [16]. In the RPGM model, each group has a center, which is either a logical center or a group leader node. The movement of the group leader determines the mobility behaviour of the entire group. Sanchez and Manzoni [18] propose a set of mobility models in which the mobile nodes travel in a cooperative manner such as Column Mobility Model, Pursue Mobility Model and Nomadic Mobility Model.

In all previous models, nodes can move freely and randomly anywhere in a simulation field. In realistic scenarios, we observe that generally, the environment of a node has specificities that can restrict node movement. In mobility models with geographic restrictions, a node may move in a pseudo-random way either because of predefined pathways on the simulation field or due to the presence of obstacles. In Pathway mobility model [19], a random graph is used to model the map of a city. The vertices of the graph represent the buildings of the city, and the edges model the streets and freeways between those buildings. Each node moves towards a randomly chosen destination through the shortest path along the edges. Similarly, in the Freeway mobility model and Manhattan mobility model [21], the movement of mobile node is also restricted to the pathways in the simulation field. In Obstacle Mobility Model, another geographic constraint is considered, obstacles.

Further mobility models can be found here[20].

\section{B. Uncertainties in Routing in Ad Hoc networks}

Although several routing protocols were proposed $[2][6][3][4]$, the majority adapt badly to specificities of the ad hoc network. They tend to choose a minimum hop path without guaranty for the choice of reliable path. To choose the most stable path is fundamental to ensure an efficient routing for highly mobile networks. However, such strategy is not typically considered in ad hoc routing. A path described as stable, must take into account the random variation in nodes states as well as their mobility which leads to link breakages. Furthermore, various delays make instants of such events often hardly known. The information on topological changes sent by a node can be out-of-date at the moment of its perception by the other nodes in the ad hoc network. Finding the most stable path must then anticipate and predict topological changes. Stability of a path is based upon the stability of all nodes composing it and all links connecting them. The stability of a node or its existence in the network at a given moment depends on several factors among them, its energy level, its power management strategy and the behavior of the user driving the node and who can choose to turn off the node whenever. Besides, a distance which separates two given nodes is variable and depends on the mobility of the two adjacent nodes what leads to the existence or the disappearance as well as the performance of the link. Consequently, we conclude that the existence of the calculated paths can be affected by some risks characterizing the random 
aspect of the ad hoc networks. We identify the existence of the following risks:

--The nodes stability : nodes of an ad hoc network can alternate periods of random and spontaneous existence and inexistence. Disappearances can be due to the collapse of energy or quite simply decided by the user piloting the mobile node. The stability of nodes affects greatly the existence of paths since if a single node of a given path comes to disappear, the whole path is broken.

--Links : links of an ad hoc network are dynamic. Link existence is controlled by the existence of its ends and their mobility. The stability of links is vital to the stability of paths since a path disappears automatically if at least one of its composing links is broken.

--The transmission delay of packages holding topological informations : can be important depending on the state of the network (congestion, interference) or the length of the path through which packets are transmitted. If the time of delivery is important, carried information can be out-of-date at the moment it is received and used by a node.

--The routing period: periodicity of the topological exchanges, in case of proactive routing protocols, imposes an information storage introducing a greater probability of error at the moment of routing. The storage period of information makes that they are even more likely to be false at the time of their use. For the reactive routing the problem arises also and in the same way. Indeed, and although a path is calculated at the time of the routing, this path could either be calculated at the moment or taken from a cache (of the node source or another intermediate node) and thus having already a certain age and a certain residual life. In all the cases a path, even if it is lately calculated, persists for certain duration. This duration will have the same effects as the routing period for proactive routing protocols.

Even mobility models are widely used to models ad hoc networks behavior, they are insufficient to model the above risks. Major studies on routing protocols performances [21] [22] [23] in ad hoc networks, consider mobility models and thus are limited to analyzing impact of random mobility on routing.

We propose in the following section, a new ad hoc network model which we called MarkovNet, modeling not only mobility behavior of nodes, but also all the identified risks.

\section{NETWORK AND ROUTING MODELLING}

In our work, we intend to define a new model for ad hoc networks which can reflect the risks that we identified previously. We aim at defining a simple model that can represent, in an abstract way, state's changes of both links and nodes regardless to their type of mobility as well as random delays of packets delivery.

In what follows, we present our model, which we called MarkovNet, then we define how we model, by MarkovNet, a simple proactive routing protocol.

\section{A. Ad Hoc Network Model: MarkovNet}

Let us consider the complete graph $\mathrm{G}=(\mathrm{V}, \mathrm{E})$ where $\mathrm{V}$ is the set of $\mathrm{N}$ vertices of the graph and $\mathrm{E}$ is the set of edges connecting the various vertices. E represents all the possible edges between each pairs of vertices and which number is: $\mathrm{N}$ $(\mathrm{N}+1) / 2$. G models an ad hoc network where each node of the network is associated to a vertex of G. An edge of G models the vicinity of two nodes i.e. if their geographical location allows them to communicate.

Since a link exists if its ends exists and are sufficiently close, an existing link will then be modelled in $G$ by an existing edge and also existing extremities vertices. The topological changes (nodes mobility and their spontaneous disappearance or appearance) can be modelled by a random alternation between the states existing and non-existent of each element of the network. To model these dynamics, we associate to each vertex and each edge of the graph an automaton. This automaton defines a two-state Markov chain: existing state (U) and non-existent state (D). For each node we associate a vertex in G. A vertex exists if the associated node has the $U$ state. For each link of the ad hoc network we associate an edge. If a link exists in the network, the corresponding edge must be $\mathrm{U}$ and the extremities vertices too.

Let $\mathrm{x}$ be an element of the random graph designing the ad hoc network. $x$ can be either an edge or a vertex. We assume that the residence time in $U$ state is governed by an exponential law having $\lambda x$ as rate. Similarly, the residence time in D state is governed by an exponential law having $\mu \mathrm{x}$ as rate. At a given moment, the ad hoc network has a particular topology and can be seen as a random graph composed of all vertices having $U$ state and all edges having $U$ state with extremity vertices also having $U$ state. This graph is dynamic and changes according to edges and vertices associated exponential laws. We denote this dynamic graph by the active graph.

The choice of the parameters $\lambda x$ and $\mu x$ reflects certain behaviour of the network. Indeed, if these parameters increase, this indicates unstable elements in time, frequently oscillating between $U$ and $D$ states. As an example, for the case of edges, great values reflect strong mobility of nodes which involves frequent changes in links states. If one of the parameters is very small, this indicates rather stable elements in $U$ or $D$ state over time. In the case of nodes, if $\lambda x$ is very small, nodes tend to disappear very seldom, even never. This case could model nodes with continuous functioning and having inexhaustible sources of energy.

In addition, we consider that delivery time of a packet over the network follows an exponential law of rate $\gamma$. The average time is inversely proportional to $\gamma$ which implies that the larger $\gamma$ is, the more the average time of propagation is small.

MarkovNet is a very simple model which goal is to show trends of certain phenomena in the ad hoc networks. In addition to its simplicity of implementation, this model offers several parameters that could give total control of the behaviour of the network. The choice of the parameters $\lambda \mathrm{x}, \mu \mathrm{x}$ 
and $\gamma$ controls the behaviour of the network in terms of mobility, stability of nodes and times of propagation.

\section{B. Routing Model}

Let us consider the framework of an ad hoc network using a proactive routing protocol. This mechanism is based on the exchange of topological messages between all nodes of the network each $\mathrm{T}$ period, in order to allow to each node to build a routing table and maintain it during a $\mathrm{T}$ period. To observe such mechanism, first we choose randomly a special node of the network which we call witness node. We fix the state of its associated vertex in graph $\mathrm{G}$ at $\mathrm{U}$ for all our simulations. Our goal is to get every $\mathrm{T}$ period the new routing table of the witness node.

To build its routing table, we acquire each period $\mathrm{T}$ the current image of the network modelled by the current active graph. We call Ip the collected topology graph of the witness node. Since in a real network, a node gets propagated topological information after a certain delay, we propose to apply Ip to the proactive routing table of witness node only after a delay $\mathrm{d}$.

To compute the new forwarding table, we use Dijkastra algorithm to get shortest paths for all possible destinations. Once the new table is created, the witness node is supposed to maintain it during all the next $\mathrm{T}$ period, before getting new updates.

We are interested in studying the accuracy of the proactive routing table over each $\mathrm{T}$ period. At this end, we fix an observation period (obs) such that obs $\leq \mathrm{T}$. Each obs, we intend to compare the routing table of witness node with instantly topological information. Hence, we compute each obs the current active graph, which we denote Ic then we study the correctness of paths given by routing table.

Processing of a simulation is described in algorithm 1 .

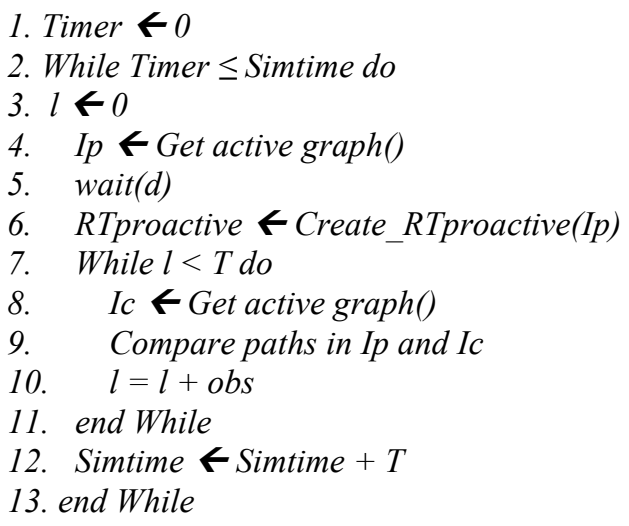

Algorithm 1. Simulation

We calculate each obs the following values based on the proactive routing table of witness node and the current active graph:

--Nc : number of accessible destinations in the current active graph.

--Np : number of accessible destinations in the proactive routing table.

--Npco : Number of destinations for which paths given by proactive routing table are still existing in the network i.e. in the current active graph and are optimal.

--Npc : Number of destinations for which paths given by proactive routing table are still existing in the current active graph but are no longer optimal i.e. shorter paths appeared.

--Npnc : Number of destinations for which paths given by proactive routing table do no longer exist in the network.

--Nnpc : Number of possible destinations in the network but unreachable in the routing table.

\section{Simulation RESUltS}

We implemented MarkovNet with proactive routing with Java programming language and using JGrapht [8] the Java graph library.

We carried out experiments on a network of 30 nodes. We choose to define a network with almost stable nodes. At this end, we assign the same $\lambda$ and $\mu$ for all vertices of the network and we fix them at 0.0001 . We assume that the packet delivery delay is deterministic and we fix it at 0.1 second. We study then both the impact of routing period increase and mobility increase on routing accuracy.

We introduce a new parameter; we call $\mathrm{c}$ which characterizes the mobility behaviour. Let $\lambda l$ and $\mu l$ be the edges parameters. We define:

$$
\begin{gathered}
\lambda 1=c \lambda 0 \\
\text { And } \\
\mu_{1}=c \mu_{0}
\end{gathered}
$$

We put: $\lambda 0=\mu 0=0.001$. We vary both $\mathrm{c}$ and the routing period $\mathrm{T}$ during several simulations which duration is $20 \mathrm{~T}$ seconds. We fix the value of obs at 1 so we can get observations each 1 second.

We plot first, the fraction of the mean number of routes given by the routing table that persist in the network during simulations and are optimal $(\mathrm{Npco} / \mathrm{Np})$. Curves are given by Figure 2. We point out that the more $\mathrm{c}$ increases, the less the fraction is. This is explained by the fact that when c increases, nodes mobility increases then routes given by the routing table tends to disappear quickly. Routing inaccuracy is more important while increasing $\mathrm{T}$ period. 


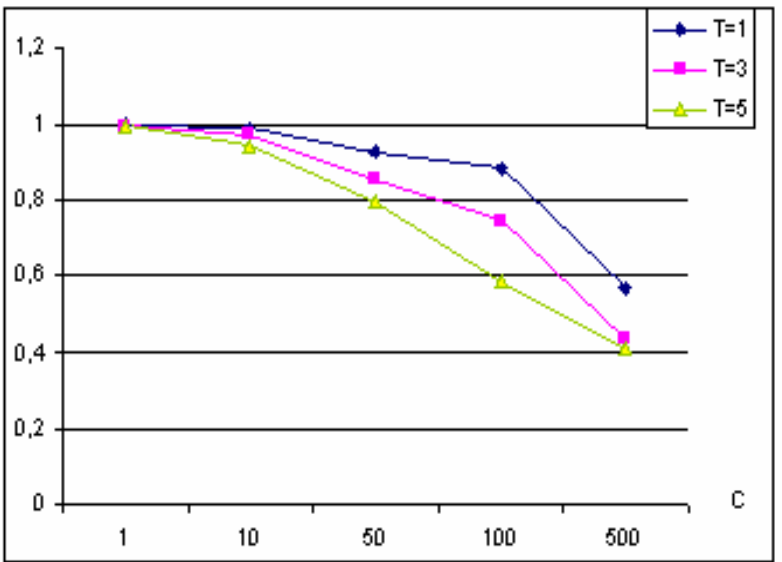

Fig. 2: Fraction of persisting and optimal routes in the routing table of witness node

In Figure 3, we plot the fraction of the mean number of routes given by the routing table but which disappeared from the network during simulations ( $N p n c / N p)$. As an effect of the first curves, we point out that the more $c$ increases, the less the fraction is. This is explained by the fact that when $c$ increases, nodes mobility increases then routes given by the routing table tends to disappear quickly. Routing inaccuracy is more important while increasing $T$ period.

In Figure 4, we plot the fraction of the mean number of destinations accessible in the network but declared non accessible by the routing process $(N n p c / N c)$. This last figure confirms also the expected behaviour of MarkovNet as an ad hoc network model. In fact, the higher the mobility is, the higher the routing information are inaccurate.

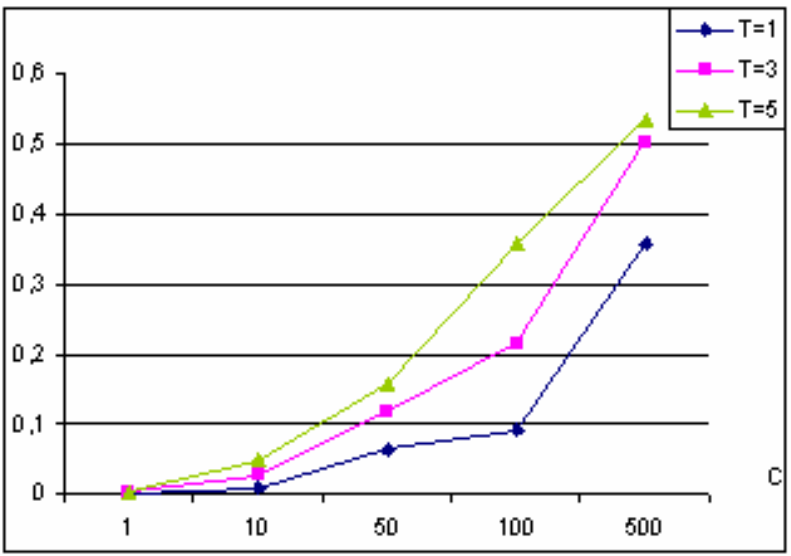

Fig. 3: Fraction of disappeared routes in the routing table of witness node

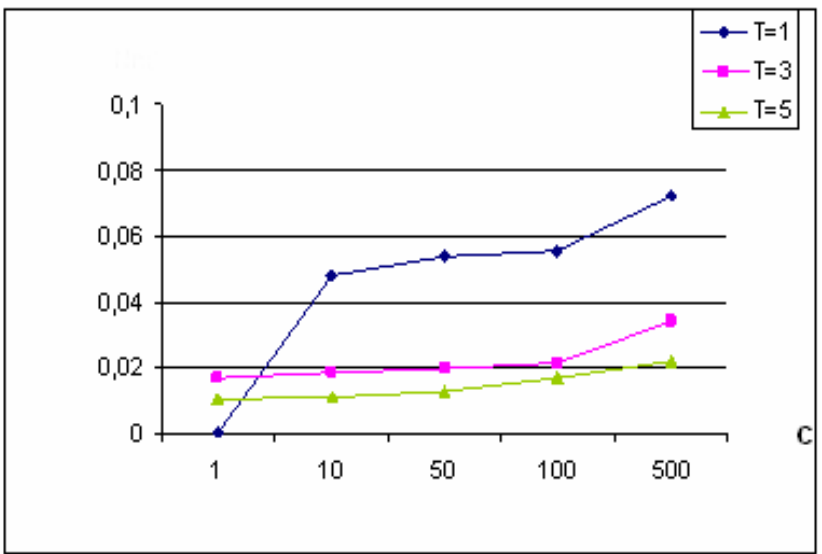

Fig. 4: Fraction of declared non accessible destinations

By the previous simulations, we notice that both mobility and high values of the routing period affect the correctness of routing decision. High mobility leads to fast disappearance of computed paths. High routing period don't let routing protocol updates in time its table which leads to expired paths but still used during routing period.

\section{CONCLUSION}

We introduced in this paper a new model for ad hoc networks which we called MarkovNet. Our model is based on the assumption that nodes and links alternate between existing and non existing states which we model by a 2-state Markov chain. As a case of study and validation for our model, we focus on the analysis of the accuracy of the proactive routing in ad hoc networks and we intended to show that our model can reproduce qualitatively the behavior of an ad hoc network while varying some parameters such as routing period.

We pointed out that the random and unpredictable dynamic behavior of ad hoc network leads uncertainties that affects the success of routing decisions. Routing period is the main parameter influencing the capability of proactive routing to adapt to network topology evolution.

We noticed through various simulations the impact of both increasing mobility nodes and routing period on the correctness of paths given by the proactive routing table. Simulations showed that the more the routing period is high, the less paths persist in the routing table. Furthermore, the number of persisting paths decrease while

increasing mobility.

Although our model is very simple, we noticed that it reproduces better than mobility mdels the behavior of an ad hoc network. We aimed at modeling states changes of both nodes and links whatever their mobility is as well as random delivery delays. Further work will be conducted to investigate the mapping between MarkovNet chain parameters and a real mobility parameters.

Furthermore, we already used MarkovNet as a test-bed to propose new routing approaches[7] to improve proactive routing protocols and help them better cope with fast and random topology changes. 


\section{REFERENCES}

[1] X. Hong, K. Xu and M. Gerla. Scalable Routing Protocols for Mobile Ad Hoc Networks. IEEE Network magazine. July/ Agust 2002. PP 1121.

[2] T. Clausen, P. Jacquet. Optimized Link State Routing Protocol. Request for Comments 3626. October 2003

[3] C. E. Perkins, E.M. Belding-Royer and Samir R. Das. Ad hoc OnDemand Distance Vector (AODV) Routing. Request for Comments 3561. July 2003.

[4] David B. Johnson, David A. Maltz, Yih-Chun Hu. The Dynamic Source Routing Protocol for Mobile Ad Hoc Networks (DSR). INTERNETDRAFT. IETF MANET Working Group. July 2004.

[5] E. M. Royer and C-K Toh. A review of the current routing protocols for ad hoc mobile wireless network. IEEE personal communication magazine. April 1999. PP 46-51.

[6] C. E.Perkins and P. Bhagwat. Highly Dynamic Destination Sequenced Distance Vector Routing (DSDV) for Mobile Computers. Proceedings of the SIGCOMM'94. PP 234-244, August 1994.

[7] R. Marie, M. Molnar and H. Idoudi. A simple automata based model for stable routing in dynamic ad-hoc networks. Submitted to MSWIM'2007. Greece. October 2007.

[8] JGraphT : Java Graph Library. On line on: http://www.jgrapht.org. Last access: 30/06/2007.

[9] A. Medina, A. Lakhina, I. Matta, J. Byers. BRITE : An approch to universal topology generation. IEEE. 2001.

[10] B. Bollobas. Random Graphs. Academic Press, London, 1985.

[11] B. Waxman. Routing of Multipoint Connections. IEEE Journal on Selected Areas in Communications, 6(9):1617-1622, December 1988

[12] R. Albert and A.-L. Barabasi. Topology of evolving networks: Local events and universality. Physical Review Letters, 85: PP 5234-5237, 2000.

[13] J. Broch, D. A. Maltz, D. B. Johnson, Y.-C. Hu, and J. Jetcheva, A performance comparison of multi-hop wireless ad hoc network routing protocols, in Proceedings of the Fourth Annual ACM/IEEE International Conference on Mobile Computing and Networking(Mobicom98), ACM, October 1998.

[14] L. Breslau, D. Estrin, K. Fall, S. Floyd, J. Heidemann, A. Helmy, P. Huang, S. McCanne, K. Varadhan, Y. Xu, and H. Yu, Advances in network simulation, in IEEE Computer, vol. 33, no. 5, May 2000, pp. 59--67.

[15] B. Liang, Z. J. Haas, Predictive Distance-Based Mobility Management for PCS Networks, in Proceedings of IEEE Information Communications Conference (INFOCOM 1999), Apr. 1999.

[16] X. Hong, M. Gerla, G. Pei, and C.-C. Chiang, A group mobility model for ad hoc wireless networks, in Proc. ACM Intern. Workshop on Modeling, Analysis, and Simulation of Wireless and Mobile Systems (MSWiM), August 1999.

[17] C. Bettstetter. Smooth is Better than Sharp: A Random Mobility Model for Simulation of Wireless Networks, in Proc. ACM Intern. Workshop on Modeling, Analysis, and Simulation of Wireless and Mobile Systems (MSWiM), Rome, Italy, July 2001.

[18] M. Sanchez and P. Manzoni, A Java-Based Ad Hoc Networks Simulator, in Proceedings of the SCS Western Multiconference Web-based Simulation Track, Jan.1999.

[19] J. Tian, J. Hahner, C. Becker, I. Stepanov and K. Rothermel. Graphbased Mobility Model for Mobile Ad Hoc Network Simulation, in the Proceedings of 35th Annual Simulation Symposium, in cooperation with the IEEE Computer Society and ACM. San Diego, California. April 2002.

[20] T. Camp, J. Boleng, and V. Davies, A Survey of Mobility Models for Ad Hoc Network Research, in Wireless Communication and Mobile Computing (WCMC): Special issue on Mobile Ad Hoc Networking: Research, Trends and Applications, vol. 2, no. 5, pp. 483-502, 2002.

[21] Fan Bai, Narayanan Sadagopan, Ahmed Helmy. IMPORTANT: A framework to systematically analyze the Impact of Mobility on Performance of RouTing protocols for Adhoc NeTworks. Infocom'03, April 1-3, 2003, San Francisco, California, USA.

[22] Maltz, D. B. Johnson, Y.-C. Hu, and J. Jetcheva, A performance comparison of multi-hop wireless ad hoc network routing protocols, in Proceedings of the Fourth Annual ACM/IEEE International Conference on Mobile Computing and Networking(Mobicom 98), ACM, October 1998
[23] F. Bai, N. Sadagopan, and A. Helmy, The IMPORTANT framework for analyzing the Impact of Mobility on Performance of Routing Protocols for Ad Hoc Networks, in AdHoc Networks Journal - Elsevier Science, Vol. 1, Issue 4, pp. 383-403, November 2003. 